\title{
A water-soluble high molecular weight substance isolated from Hyuganatsu orange (Citrus tamurana), suspected to be a polysaccharide, inhibits rat osteoclast cell formation
}

\section{Hiroko Hata $^{1}$, Masatoshi Yamaguchi ${ }^{1}$, Hiroshi Sameshima ${ }^{1}$, Tsuyomu Ikenoue ${ }^{1}$, Junko Matsubara $^{2}$, Makoto Tsuboi ${ }^{2}$, and Takashi Tanaka ${ }^{3}$}

${ }^{1}$ Department of Obstetrics and Gynecology, Faculty of Medicine, University of Miyazaki, 5200 Kihara, Kiyotake-Cho, Miyazaki City, Miyazaki 889-1692, Japan; ${ }^{2}$ Ichimaru Phalcos Company, 318-1, Asagi, Motosu City, Gifu 501-0475, Japan; ${ }^{3}$ Laboratory of Natural Product Chemistry, Nagasaki University Graduate School of Biomedical Sciences, 1-14 Bunkyo-cho, Nagasaki City, Nagasaki 852-8521, Japan

Corresponding author: Masatoshi Yamaguchi, Professor, Department of Obstetrics and Gynecology, Faculty of Medicine, University of Miyazaki, 5200 Kihara, Kiyotake-Cho, Miyazaki City, Miyazaki 889-1692, Japan

Submission date: March 2, 2015; Acceptance date: May 31, 2015: Publication date: June 1, 2015

\begin{abstract}
Background: Osteoporosis is detrimental to aged women's health care. We previously reported that Hyuganatsu orange (Citrus Tamurana) contains active substances that inhibit osteoclast activities. Prior to conducting a human study, we sought to identify the biological active substance in the Hyuganatsu orange which suppresses osteoclast formation.
\end{abstract}

Methods: We isolated five fractions from a Hyuganatsu orange extract according to molecular weight. Each fraction was tested to determine its suppressive effect on the formation of osteoclasts in rats. We also used high-performance liquid chromatography (HPLC), infra-red (IR), and ${ }^{1} \mathrm{H}$ and ${ }^{13} \mathrm{C}$ NMR spectroscopy to evaluate its chemical structure. Data was recorded as mean \pm standard error of the mean. The Mann-Whitney test was used, and a p-value of <.05 was considered statistically significant.

Results: The highest and lowest molecular weight fractions showed significant suppression activity on rat osteoclast formation $(\mathrm{p}<.05)$. The lowest molecular weight fraction was identified as hesperidin using thin layer chromatography. Additionally, IR absorption revealed that the highest molecular weight fraction was not a flavonoid. With regard to chemical structure, ${ }^{1} \mathrm{H}$ and ${ }^{13} \mathrm{C}$ NMR spectroscopy suggested that the highest molecular weight fraction had signals compatible with a polysaccharide such as galacturonic acid.

Conclusions: Hyuganatsu orange contains a biological active substance other than hesperidin 
that may be a polysaccharide and may suppress osteoclast formation.

Key words: Citrus Tamurana, TRAP positive cell, Polysaccharides, Rat osteoclast cell, in vitro study

\section{BACKGROUND}

Osteoporosis is detrimental to aged women's health care, a progressive bone disease characterized by a decrease in bone mass, density, and bone quality, all of which lead to an increased risk of fracture [1]. Bone mass and density maintain a balance between bone formation and bone absorption. While bone formation is partly related to osteoblast activity, bone absorption is related to osteoclast activity. Before menopause, estrogen helps control osteoblast and osteoclast levels within women; after menopause, decreased estrogen levels within women are the primary etiology for osteoporosis. Ideally, treatment with estrogen is effective for osteoporosis [2]. However, long-term administration of estrogen can cause some unfavorable effects, such as increased risks for mammary cancer [3]. In addition to estrogen, other medications also suppress osteoclast formation, with only teriparatide stimulating osteoblast formation [4]. However, these drugs cannot increase bone density in young women after bone density has decreased to osteoporotic levels. Thus, it is important to develop prevention measures against osteoporosis.

The Hyuganatsu Orange (Citrus tamurana) is a citrus fruit originating in Miyazaki Prefecture. In previous studies, we have reported the presence of a substance that inhibits the production of rat osteoclast cells in C. Tamurana [5]. It is possible that this active substance we found has various biological activities, such as the promotion of fibroblast migration [6].

Oranges are rich in flavonoid, which suppresses osteoclast formation. In our Hyuganatsu experiments, we discovered that Hesperidin, a flavonoid, may be responsible for suppressing osteoclast formation. However, our pilot study showed that the Hyuganatsu orange has a relatively low level of hesperidin compared to the mandarin orange. Therefore, we performed this study to identify other active substances in the Hyuganatsu orange which affect osteoclast formation.

\section{MATERIALS AND METHODS}

Based on a previous study [5], we isolated the water-soluble fraction of $C$. tamurana with slight modifications (Figure 1). We modified methyl alcohol extraction to ethyl alcohol extraction for the procedure, before extracting the residue with water. The reason for this modification is that methanol extraction cannot be used for food, due to potential toxicity for human. We achieved fractionation of the aqueous extract according to the molecular weight; gel filtration was performed in a Sephadex G25 column. Immediately after the void volume was identified, the first fraction was designated as fraction 1. In total, 5 fractions were collected using water as an elution solution. Each fraction was evaporated and dried, before being developed using a thin layer chromatography plate manufactured by Merck (TLC Silica gel 60 F254, $20 \mathrm{~cm}$ x $20 \mathrm{~cm}$ ). The developing solvent was a mixture of butanol, acetic acid, and water in a 4:1:2 ratio, respectively, and the spots were visualized by spraying $20 \%$ sulfuric acid. Standard hesperidin was purchased from Nacalai Tesque Co. Ltd. (Kyoto, Japan). 


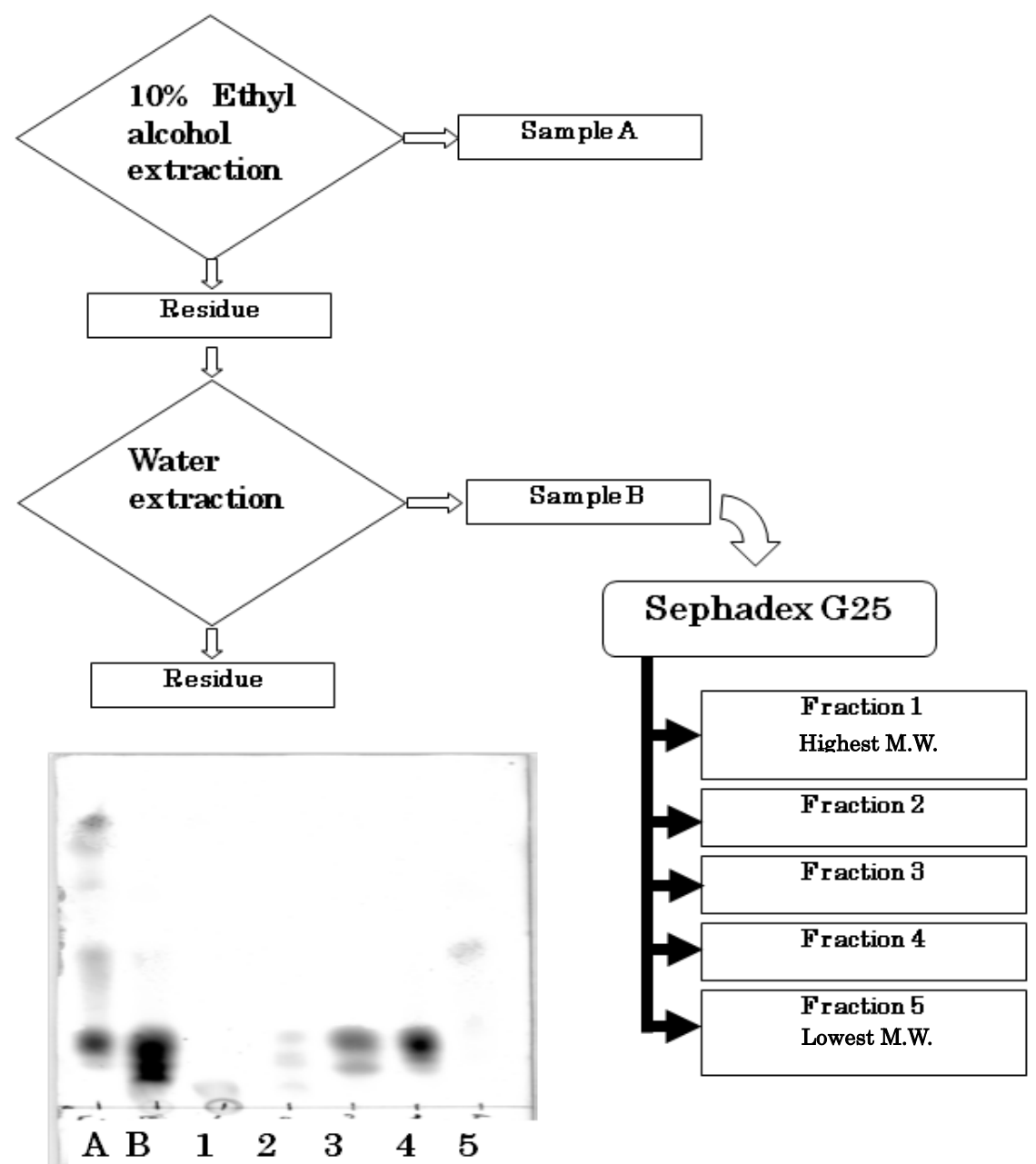

Figure 1. Brief extraction procedure and thin layer chromatography. Hyuganatsu orange extracts were obtained with $10 \%$ ethyl alcohol, followed by water extraction. Water extract was applied to a Sephadex G25 column and fractionated according to molecular weight. Each sample was analyzed with thin layer chromatography. A: sample A (extraction with $10 \%$ ethyl alcohol); B: sample B (water extraction) 1-5: fractions of Sephadex G25 chromatography.

Additionally, the molecular weight of the largest fraction (fraction 1) was examined with HPLC. TSKgel G5000-PWXL ( $\varphi 7.8 \mathrm{~mm} \times 300 \mathrm{~mm}$ ) was manufactured by Tosoh (Tokyo, Japan), using a Shimadzu LC-20AD system along with a pullulan marker. Detection was performed using a parallax refractometer (RID-10A) manufactured by Shimadzu, Kyoto, Japan. A moving bed method was used with a phosphoric acid buffer solution of $\mathrm{pH} 6.8$, with a flow rate of $0.5 \mathrm{~mL} / \mathrm{min}$ and a column temperature of $40^{\circ} \mathrm{C}$.

The highest molecular weight fraction (fraction 1) was also analyzed by HPLC, infra-red (IR), and ${ }^{1} \mathrm{H}$ and ${ }^{13} \mathrm{C}$ NMR spectroscopy. HPLC was performed on a Cosmosil $5 \mathrm{C}_{18}$-ARII (Nacalai Tesque, Kyoto, Japan) column $(250 \times 4.6 \mathrm{~mm}$ i.d., $5 \mu \mathrm{m})$, with gradient elution from $4 \%$ to $30 \%$ (39 $\mathrm{min}$ ) and from $30 \%$ to $75 \%$ (15 min) $\mathrm{CH}_{3} \mathrm{CN}$ in $50 \mathrm{mM} \mathrm{H}_{3} \mathrm{PO}_{4}$ (flow rate, 0.8 $\mathrm{mL} / \mathrm{min}$; temperature, $35^{\circ} \mathrm{C}$ ). Detection was performed with a JASCO photodiode array detector MD-2010 [JASCO Co., Tokyo, Japan]). IR spectra were obtained with a JASCO FT/IR-410 spectrophotometer (JASCO Co., Tokyo, Japan). The NMR spectra were measured on a JEOL 
JNM-AL400 (400 MHz for ${ }^{1} \mathrm{H}$ and $100 \mathrm{MHz}$ for ${ }^{13} \mathrm{C}$ NMR) spectrometer (JEOL Ltd., Tokyo, Japan).

Using a rat osteoclast cell culture kit from Primary Cell (COSMO BIO Co., Ltd.), an osteoclast progenitor cell was cultured in a 96-well plate. For each fraction, a final concentration of $0.1 \mathrm{mg} / \mathrm{mL}$ or $0.5 \mathrm{mg} / \mathrm{mL}$ was ensured. In our pilot study, we tested culture experiment in $0.5 \mathrm{mg} / \mathrm{mL}$. In this concentration, we observed significant difference in TRAP positive cells. Therefore, we tried culture study in $0.5 \mathrm{mg} / \mathrm{mL}$ and $0.1 \mathrm{mg} / \mathrm{mL}$. The culture medium consisted of $10 \%$ fetal bovine serum, $\alpha-\mathrm{MEN}$ culture medium containing $50 \mathrm{ng} / \mathrm{mL}$ of $\mathrm{M}-\mathrm{CSF}$, and $15 \mathrm{ng} / \mathrm{mL}$ of Receptor activator of nuclear kappa-B ligand (RANKL). Culturing was performed for 5 days. Staining of the formed osteoclast cells was achieved using a tartrate-resistant acid phosphate (TRAP) staining kit by Primary Cell (COSMO BIO co., ltd.). TRAP-positive cells were directly counted using a microscope. Red colored polynucleic cell was identified as TRAP positive cell. The Mann-Whitney test was used to analyze differences in TRAP-positive cells from the culture. Data results are expressed as mean \pm standard error of the mean, and $p<.05$ was considered statistically significant.

\section{RESULTS}

From $6.0 \mathrm{~g}$ of the sample B (water extracts), $0.12 \mathrm{~g}$ of fraction $1(2 \%), 0.45 \mathrm{~g}$ of fraction $2(7.5 \%)$, $4.83 \mathrm{~g}$ of fraction $3(80.5 \%), 0.32 \mathrm{~g}$ of fraction $4(5.3 \%)$ and $0.19 \mathrm{~g}$ of fraction $5(3.2 \%)$ were isolated. Photographs of the typical culture well of the control well and each fraction are presented in figure 2.

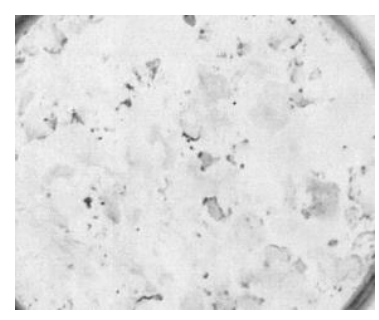

a) Control

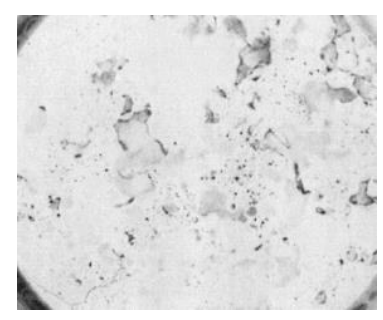

e) Fraction 4

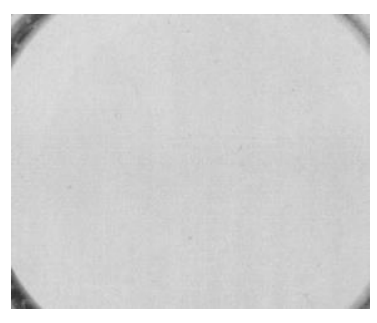

b) Fraction 1

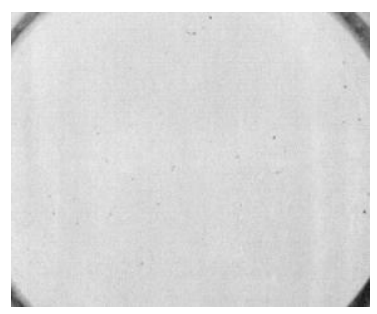

f) Fraction 5

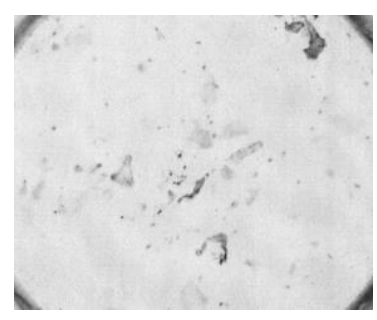

c) Fraction 2

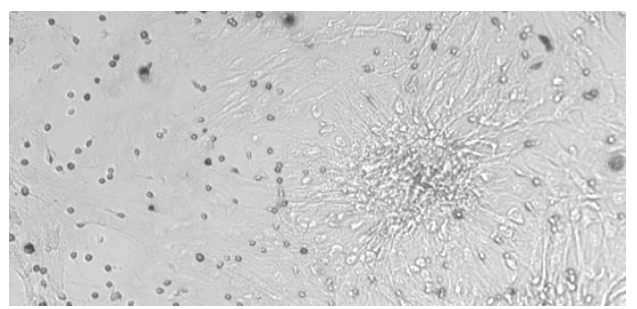

g) High magnification

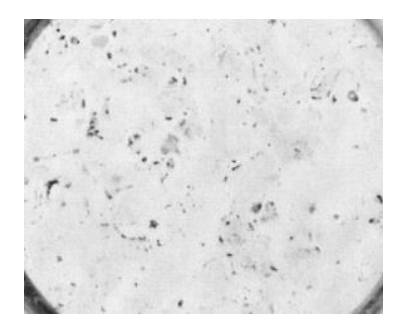

d) Fraction3

Figure 2. Typical photograph of the culture well of the control well (a), treated with fraction 1 (b), fraction 2 (c), fraction 3 (d), fraction 4 (e), and fraction 5 (f). High magnification photograph of the fraction 1 was presented in $(\mathrm{g})$. Note preosteoclast cell treated with fraction 1 changed to spindle shape and cell fusion was not observed. Only a few TRAP positive cells were found.

Precursor cell of the osteoclast treated with fraction 1 had a spindle like shape and cell 
fusion was not observed (figure $2 \mathrm{~g}$ ). The results of osteoclast cell formation for each fraction are presented in Figure 3. Since the osteoclast cell number in each well of the control group was different in each series of experiments (range, 25-64 cells), results were expressed as a percentage of the average number of control wells. TRAP positive cells in wells treated with 0.1 $\mathrm{mg} / \mathrm{mL}$ in each fraction was $41.7 \% \pm 6.1 \%$ in fraction $1 ; 91.5 \% \pm 4.9 \%$ in fraction $2 ; 105.2 \% \pm$ $6.4 \%$ in fraction $3 ; 94.5 \% \pm 6.7 \%$ in fraction 4 ; and $62.0 \% \pm 8.6$ in fraction 5 . The cell number value in cells treated with $0.5 \mathrm{mg} / \mathrm{mL}$ in each fraction was $3.0 \% \pm 0.9 \%$ in fraction $1 ; 45.9 \% \pm$ $7.4 \%$ in fraction $2 ; 96.6 \% \pm 8.1 \%$ in fraction $3 ; 104.3 \% \pm 7.4 \%$ in fraction 4 ; and $2.15 \pm 0.7 \%$ in fraction 5. We found that fractions 1 and 5 had the strongest inhibitory action on osteoclast cell formation $(\mathrm{p}<.05)$, whereas fraction 2 had the weak inhibitory action. And fraction 3 and 4 had no inhibitory effect (Figure 3).

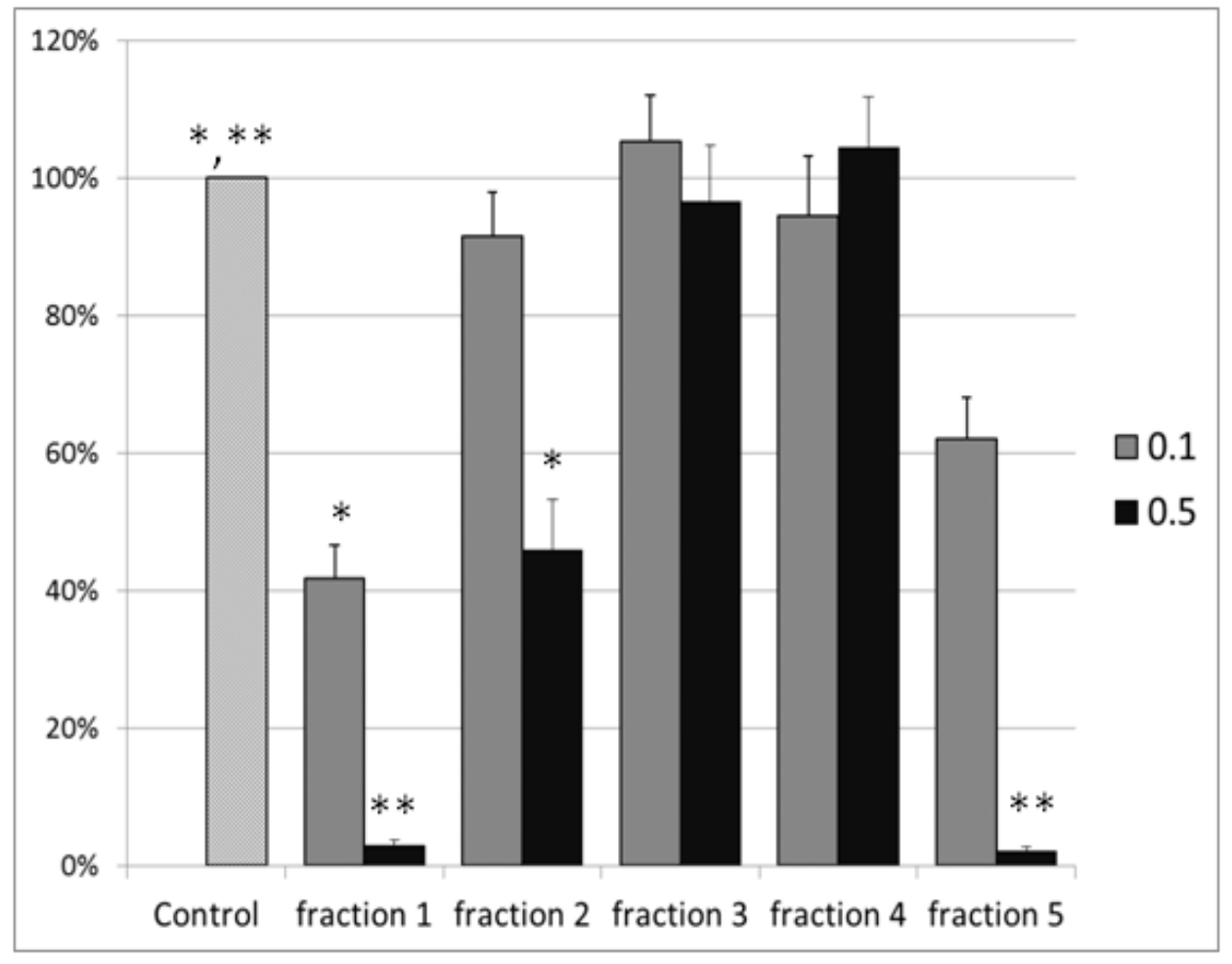

Figure 3. TLC pattern of fraction 5. Fraction 5 was developed in lane A and standard hesperidin was shown in lane C. Mixture of the fraction 5 and standard hesperidin was developed in lane B. In standard lane, two bands (indicated by arrow) were observed. Lower arrow indicates degradation product of the hesperidin. Main band was identical in fraction 5 and standard hesperidin. Therefore, we concluded that fraction 5 mainly consisted of hesperidin.

Thin layer chromatography results (Figure 1) indicated that fraction 1 did not move from its origin, demonstrating a relatively high molecular weight. Since the RF value of fraction 5 was identical to the RF value of commercially available standard hesperidin, the lowest molecular weight fraction was interpreted as hesperidin (Figure 4). The molecular weight of fraction 1 was approximately $68 \mathrm{kDa}$.

HPLC analysis of fraction 1 showed no peaks, thereby suggesting that the substance does 
not absorb UV light (Figure 3). Moreover, the ${ }^{1} \mathrm{H}-\mathrm{NMR}$ spectrum did not demonstrate aromatic proton signals. In fact, large broad signals of oxygenated methine and methylene protons were observed in the $\delta$ range of 3.0-5.0, which can be attributed to sugar protons (upper panel of Figure 4).

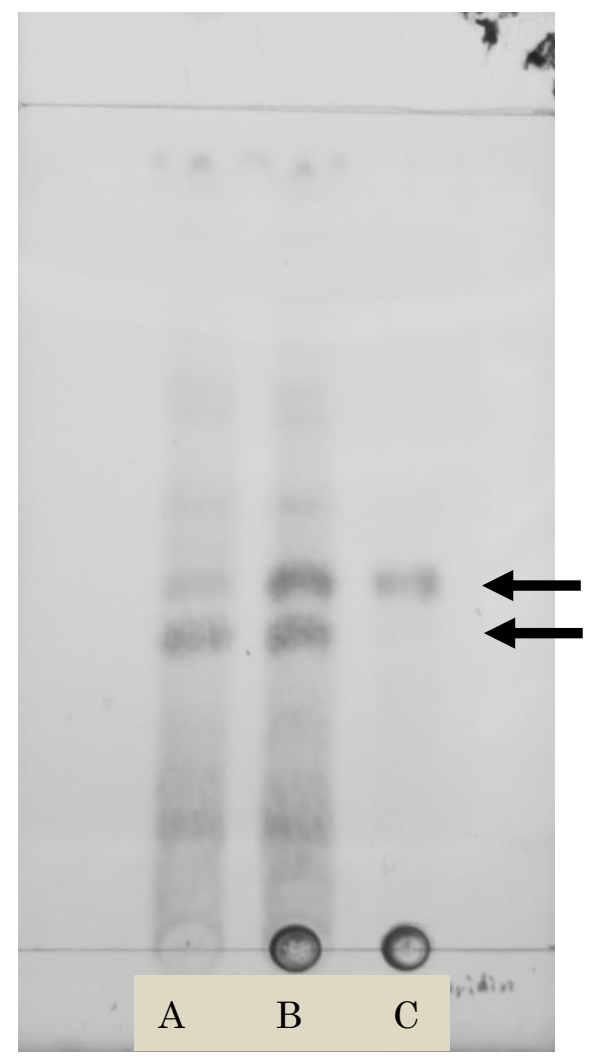

Figure 4. Number of the tartrate positive acid phosphatase (TRAP)-positive cells. Since the number of the TRAP-positive cells in the control (medium without Hyuganatsu orange extract) well was not same in each culture plate, the number was expressed as a percentage of the mean value of the control wells. The values from each column were expressed as a mean \pm standard error of the mean. Fractions 1 and 5 significantly suppressed TRAP-positive cells. Fraction 5 was identified as hesperidin. The asterisks indicate significant differences $(* \mathrm{p}<.05$ and $* * \mathrm{p}<.01)$ when compared with the control wells. The final concentration of the fraction was $0.1 \mathrm{mg} / \mathrm{mL}$ (gray bar) or $0.5 \mathrm{mg} / \mathrm{mL}$ (dark bar).

The presence of sugar moieties were additionally confirmed by ${ }^{13} \mathrm{C}$ NMR spectroscopic analysis (lower panel of Figure 4), which exhibited anomeric carbon signals at a $\delta$ value of 110.3, 108.6, and 104.5 together with complex signals, due to oxygenated aliphatic carbons in the $\delta$ range of 62.4-84.9. Signals attributable to flavonoids were not observed in the spectrum. In addition, signals in the $\delta$ range of 165-175 suggested the presence of uronic acid moieties, such as galacturonic acid, in the molecules. This was supported by the appearance of a strong absorption at $1,658 \mathrm{~cm}^{-1}$, which may be attributed to the COO-group in the IR spectrum and also absorptions at $3,410 \mathrm{~cm}^{-1}$ and $1,075 \mathrm{~cm}^{-1}$ assignable to the hydroxyl groups. 

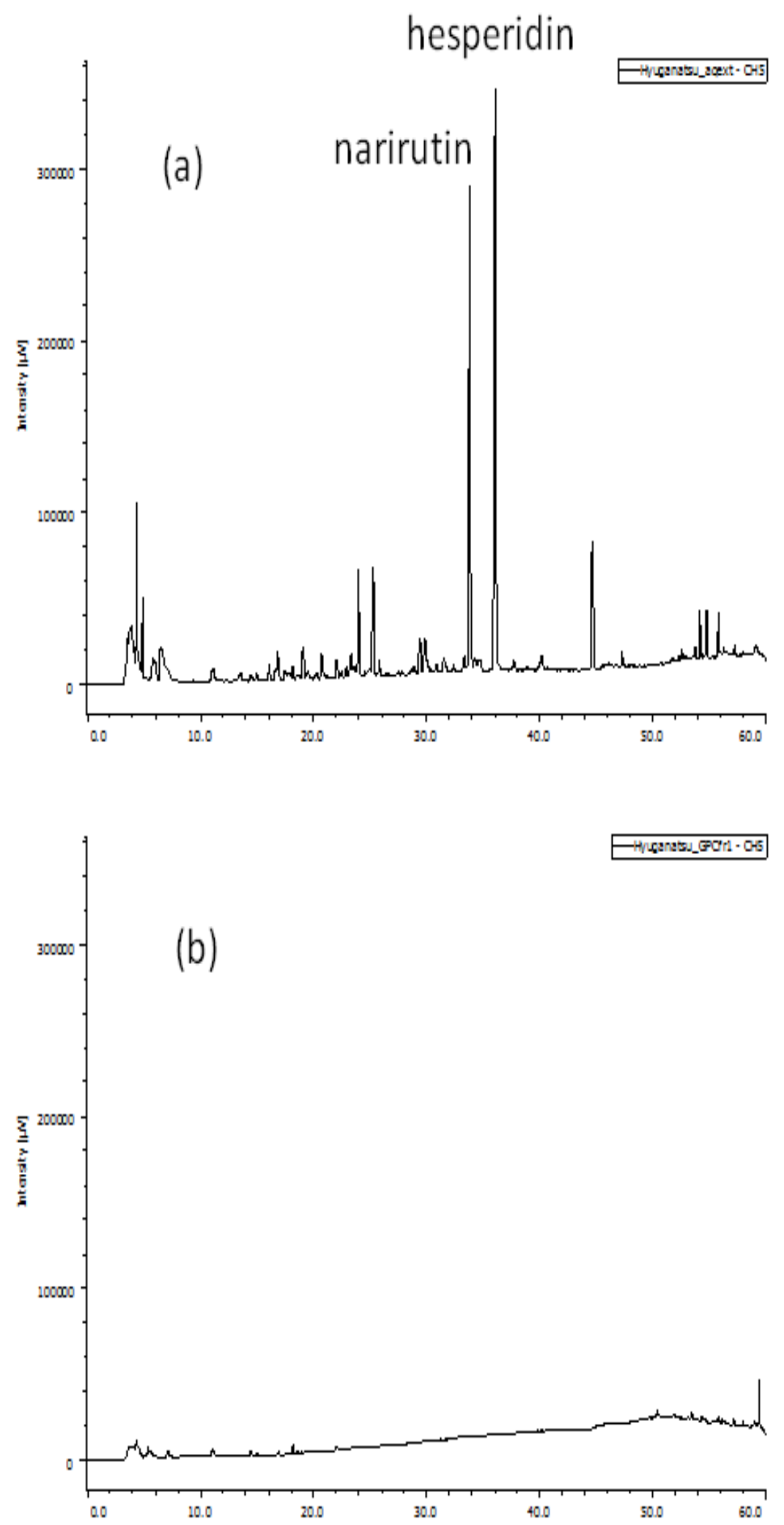

Figure 5. High-performance liquid chromatography elution pattern of the water extract (a, upper panel, sample B of the figure one) and fraction 1 (b, lower panel). A sharp UV peak of hesperidin and narirutin was observed in sample B. However, no peak was noted in fraction 1 


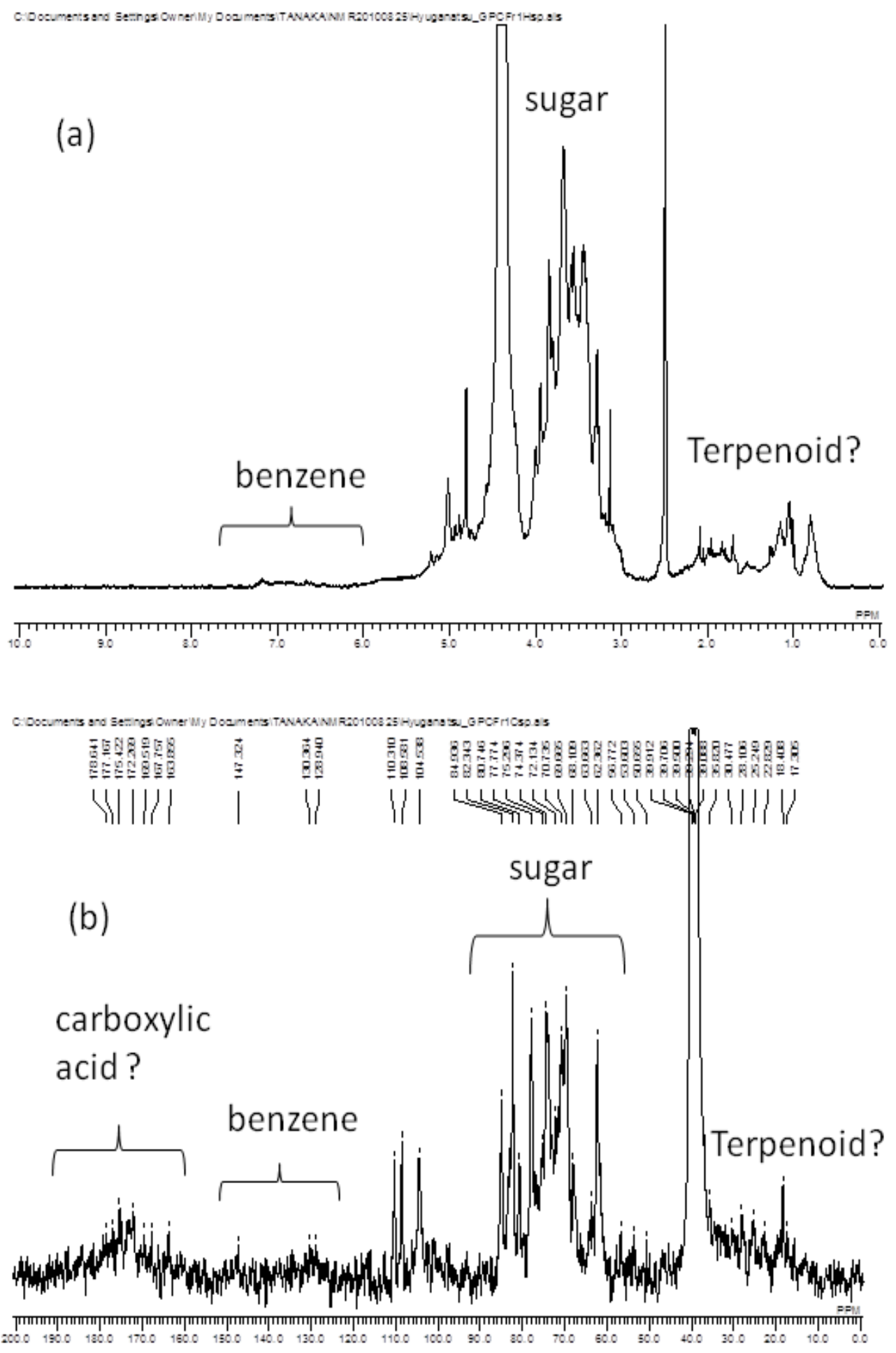

Figure 6. The ${ }^{1} \mathrm{H}-\mathrm{NMR}$ (a, upper panel) and ${ }^{13} \mathrm{C}-\mathrm{NMR}$ spectra (b, lower panel) of fraction 1. There was no benzene signal in the ${ }^{1} \mathrm{H}-\mathrm{NMR}$ or ${ }^{13} \mathrm{C}-\mathrm{NMR}$ spectra. Note that the signals are attributable to sugar residues, including uronic acid moieties.

\section{DISCUSSION}

Osteoporosis is a disease which significantly affects the elderly, often causing them to become bedridden, an increasingly problematic disease afflicting aging populations. A large number of 
medications have been developed for the treatment of this disease. However, since drugs such as SERMs have side-effects, natural food products that have low risk of side-effects may be beneficial [7]. Functional food products can be separated into those that enhance various functions and those that reduce risk [8]. A significant number of functional foods have been reported to be effective against osteoporosis. These products include dried fruits (plums) [9, 10], water-soluble extracts (Agrimonia pilosa Ledeb) [11], Greek flora [7], soybean products [12], and ethanol extracts (Japanese arrowroot) [13]. Soy isoflavones are well known, an active ingredient in soya beans $[12,14]$. There have been reports that soy isoflavones have a similar action to SERMs [14, 15], although other reports have also found that these are not effective [16].

An increase in bone mineral density in vivo is one of the effects of hesperidin on bone metabolism [17-21]. However, its main mechanism of action involves an inhibitory action on bone resorption [17-21]. Therefore, it seems unlikely that hesperidin would have a great effect on bone formation [18]. In vitro studies have demonstrated that hesperidin inhibits osteoclast cell formation [21-23], without any effect on osteoblast proliferation [24]; however, hesperidin also promotes differentiation from osteoblast progenitor cells to osteoblast cells [24-26]. We found that the examined substance (fraction 1 in this study) differs from hesperidin on a number of points. Significantly, the biggest difference is the molecular weight. The molecular weight of the fraction 1 was approximately $68 \mathrm{kDa}$, and therefore cannot be hesperidin (molecular weight, approximately $610 \mathrm{Da}$ ). Moreover, there was no UV absorption in HPLC analysis, suggesting the absence of a benzene structure in fraction 1 . Finally, ${ }^{13} \mathrm{C}$ NMR also could not detect any benzene structure motif. Based on these findings, we concluded that high molecular weight fraction is not hesperidin or a related compound.

Alternatively, NMR spectroscopic analysis on fraction 1 suggested that the active substance is a polysaccharide. In addition, ${ }^{13} \mathrm{C} N \mathrm{NMR}$ and IR spectra indicated the presence of carboxyl groups. Based on these findings, we concluded that the water-soluble, highest molecular weight fraction is a polysaccharide which may include or be similar to pectin or pectic acid with certain modifications. However, it is difficult to identify chemical structures from water-soluble substances. Nonetheless, there are several investigations which indicate that pectin or modified pectin may have anti-cancer metastasis effects [27-30]. For example, Brouns et al [31] reported the cholesterol-lowering properties of pectin. These effects suggest that there are new biological functions of the citrus polysaccharide, which may include pectin or related compounds.

Based on the thin layer chromatography results, we noted that hesperidin was still present in the water-soluble extracts. Therefore, the inhibitory action we previously reported on osteoclast formation was likely due to a combination of residual hesperidin and another undetermined high molecular weight substance.

Weak suppression effects were observed in fraction 2. This fraction show similar IR spectra pattern from the previous experiment with fraction 1. The difference of these two fraction also indicates similar molecular weight as fraction 1. As a result, we hypothesize that fraction 2 is a degradation or digested products of fraction 1.

Since fraction 3 and 4 have the tendency of stimulating TRAP positive cells, one may question the safety of these Hyuganatsu extracts. However, as we could not find significant difference in TRAP positive cell numbers, we concluded fraction 3 and 4 have no effect to TRAP positive cell. In this study, we did not stain Cathepsin K. Cathepsin K is an important marker for 
verifying osteoclast. Nevertheless, Cathepsin K staining is not always essential in culture study. Therefore, we concluded that the high molecular weight Hyuganatsu extract suppressed, at the very least, TRAP positive cells.

\section{CONCLUSIONS}

In conclusion, we found that $C$. tamurana contains a substance that is not a flavonoid, and inhibits rat osteoclast progenitor cells from differentiating into osteoclasts. Since Hyuganatsu extracts are obtained from natural foods, we presume that the intake of the extracts may not be harmful and potentially effective for postmenopausal women.

List of Abbreviations used: High-performance liquid chromatography (HPLC), Infra-Red (IR), Nuclear magnetic resonance (NMR), Ultra violet (UV), Selective estrogen receptor modulators (SERMs), Tartrate-resistant acid phosphate (TRAP)

Competing Interests: The authors declare that they have no competing interests.

Author's Contributions: Hiroko Hata, MD is the principle investigator for this study providing cell culture. She wrote this manuscript.

Masatsohi Yamaguchi, MD is the corresponding author of this manuscript. He wrote the grant proposal and contributed fundamental conceptualization. Hiroshi Sameshima, MD and Tsuyomu Ikenoue, MD are department heads. They contributed fundamental conceptualization. Junko Matsubara and Makoto Tsuboi, PhD are staff of the Ichimaru Falcos Company. They performed gel filtration and provided high molecular fraction of Hyuganatsu orange extracts. They also analyzed this fraction with TLC Takashi Tanaka, PhD performed Spectroscopy (NMR) of the high molecular fraction of Hyuganatsu orange extracts

Acknowledgments and Funding: This investigation was supported in part by Grant in Aid from Science and technology research promotion program for agriculture, forestry, fisheries and food industry (25086C).

\section{REFERENCES}

1. Consensus development conference: Prophylaxis and treatment of osteoporosis. Am J Med 1991; 90:107-110.

2. Hernberg CA. Treatment of postmenopausal osteoporosis with estrogens and androgens. Acta Endocrinol (Copenh) 1960; 34: 51-59.

3. Roehm E. Hormone therapy use and outcomes in the Women's Health Initiative trials. JAMA 2014; 311: 417.

4. Vescini F, Grimaldi F. PTH 1-84: bone rebuilding as a target for the therapy of severe osteoporosis. Clin Cases Miner Bone Metab 2012; 9: 31-36.

5. Yamaguchi M, Sameshima H, Ikenoue T, Tsuboi M, Hidaka M, Arimori K. Hyuganatsu orange (Citrus tamurana Hort. Ex Tanaka) contains a water soluble substance that suppresses bone loss in ovariectomized rats. Biosci Biotechnol Biochem 2012; 76: 364- 
367.

6. Harishkumar M, Yamaguchi M, Sameshima H, Ikenoue T, Maruyama M. Revealing the mechanism of in vitro wound healing properties of Citrus tamurana extract. Biomed Res Int 2013; 2013: 963457.

7. Kassi E, Papoutsi Z, Fokialakis N, Messari I, Mitakou S, Moutsatsou P. Greek plant extracts exhibit selective estrogen receptor modulator (SERM)-like properties. J Agric Food Chem 2004; 52: 6956-6961.

8. Roberfroid MB. Functional foods: concepts and application to inulin and oligofructose. Br J Nutr. 2002; 87: S139-S143.

9. Arjmandi BH, Johnson CD, Campbell SC, Hooshmand S, Chai SC, Akhter MP. Combining fructooligosaccharide and dried plum has the greatest effect on restoring bone mineral density among select functional foods and bioactive compounds. J Med Food 2010; 13: 312-319.

10. Hooshmand S, Arjmandi BH. Viewpoint: dried plum, an emerging functional food that may effectively improve bone health. Ageing Res Rev 2009; 8: 122-127.

11. Lee YM, Kim JB, Bae JH, et al. Estrogen-like activity of aqueous extract from Agrimonia pilosa Ledeb. In MCF-7. BMC Complement Altern Med 2012; 12: 260.

12. Chen KI, Erh MH, Su NW, Liu WH, Chou CC, Cheng KC. Soyfoods and soybean products: from traditional use to modern applications. Appl Microbiol Biotechnol 2012; 96: 9-22.

13. Tanaka T, Tang H, Yu F, et al. Kudzu (Pueraria lobata) vine ethanol extracts improve ovariectomy-induced bone loss in female mice. J Agric Food Chem 2011; 59: 1323013237.

14. Simons R, Gruppen H, Bovee TF, Verbruggen MA, Vincken JP. Prenylated isoflavonoids from plants as selective estrogen receptor modulators (phytoSERMs). Food Funct 2012; 3: 810-827.

15. Song WO, Chun OK, Hwang I, et al. Soy isoflavones as safe functional ingredients. $J$ Med Food 2007; 10: 571-580.

16. Lagari VS, Levis S. Phytoestrogens and bone health. Curr Opin Endocrinol Diabetes Obes 2010; 17: 546-553.

17. Chiba H, Kim H, Matsumoto A, et al. Hesperidin prevents androgen deficiency-induced bone loss in male mice. Phytother Res 2013; 28: 289-295.

18. Habauzit V, Sacco SM, Gil-Izquierdo A, et al. Differential effects of two citrus flavanones on bone quality in senescent male rats in relation to their bioavailability and metabolism. Bone 2011; 49: 1108-1116.

19. Habauzit V, Nielsen IL, Gil-Izquierdo A, et al. Increased bioavailability of hesperetin-7-glucoside compared with hesperidin results in more efficient prevention of bone loss in adult ovariectomised rats. Br J Nutr 2009; 102: 976-84.

20. Horcajada MN, Habauzit V, Trzeciakiewicz A, et al. Hesperidin inhibits ovariectomized-induced osteopenia and shows differential effects on bone mass and strength in young and adult intact rats. J Appl Physiol 2008; 104: 648-654.

21. Chiba H, Uehara M, Wu J, et al. Hesperidin, a citrus flavonoid, inhibits bone loss and decreases serum and hepatic lipids in ovariectomized mice. J Nutr 2003; 133: 1892- 
1897.

22. Ahmadi A, Hosseinimehr SJ, Naghshvar F, Hajir E, Ghahremani M. Chemoprotective effects of hesperidin against genotoxicity induced by cyclophosphamide in mice bone marrow cells. Arch Pharm Res 2008; 31: 794-797.

23. Yamaguchi M, Hamamoto R, Uchiyama S, Ishiyama K. Effects of flavonoid on calcium content in femoral tissue culture and parathyroid hormone-stimulated osteoclastogenesis in bone marrow culture in vitro. Mol Cell Biochem 2007; 303: 83-88.

24. Trzeciakiewicz A, Habauzit V, Mercier S, et al. Hesperidin stimulates differentiation of primary rat osteoblasts involving the BMP signaling pathway. J Nutr Biochem 2010; 21: 424-431.

25. Trzeciakiewicz A, Habauzit V, Mercier S, et al. Molecular mechanism of hesperetin-7-O-glucuronide, the main circulating metabolite of hesperidin, involved in osteoblast differentiation. J Agric Food Chem 2010; 58: 668-675.

26. Choi EM, Kim YH. Hesperidin attenuates the highly reducing sugar-triggered inhibition of osteoblast differentiation. Cell Biol Toxicol 2008; 24: 225-231.

27. Hossein G, Keshavarz M, Ahmadi S, Naderi N. Synergistic effects of PectaSol-C modified citrus pectin an inhibitor of Galectin-3 and paclitaxel on apoptosis of human SKOV-3 ovarian cancer cells. Asian Pac J Cancer Prev 2013; 14: 7561-7568.

28. Hao M, Yuan X, Cheng H, et al. Comparative studies on the anti-tumor activities of high temperature- and pH-modified citrus pectins. Food Funct 2013; 4: 960-971.

29. Ramachandran C, Wilk BJ, Hotchkiss A, Chau H, Eliaz I, Melnick SJ. Activation of human T-helper/inducer cell, T-cytotoxic cell, B-cell, and natural killer (NK)-cells and induction of natural killer cell activity against K562 chronic myeloid leukemia cells with modified citrus pectin. BMC Complement Altern Med 2011; 11: 59.

30. Glinsky VV, Raz A. Modified citrus pectin anti-metastatic properties: one bullet, multiple targets. Carbohydr Res 2009; 344: 1788-1791.

31. Brouns F, Theuwissen E, Adam A, Bell M, Berger A, Mensink RP. Cholesterol-lowering properties of different pectin types in mildly hyper-cholesterolemic men and women. Eur J Clin Nutr 2012; 66: 591-599. 